\title{
Reducing periorbital edema and ecchymosis to a minimum when performing rhinoplasty without splints or packing
}

André Camirand MD, Jocelyne Doucet RN

Montreal, Quebec

A Camirand, J Doucet. Reducing periorbital edema and ecchymosis to a minimum when performing rhinoplasty without splints or packing. Can J Plast Surg 1995;3(4):196-198. In view of the discomfort and medical risks associated with conventional rhinoplasties, an innovative approach to aesthetic modification of the nose, which does not require splints or packing and helps to minimize risks and complications, has been developed. The steps in this procedure are presented, together with the results from nearly 200 rhinoplasties.

Key Words: Ecchymosis, Periorbital edema, Rhinoplasty

Réduction de l'oedème péri-orbitaire et de l'ecchymose à un minimum dans la rhinoplastie sans attelle ni paquetage

RÉSUMÉ : Compte tenu de l'inconfort et des risques médicaux associés aux rhinoplasties classiques, une approche novatrice a été applique à la rhinoplastie esthétique du nez; elle ne requiert ni attelle ni paquetage et contribue à minimiser les risques et les complications. Les étapes de cette intervention sont présentées, de même que les résultats de près de 200 rhinoplasties.

During training we are taught to immobilize the bones of the nose after performing an osteotomy. A pliable metal splint or a thin cast is fixed to mobile skin and then attached with stretchable Elastoplast. Packing is inserted into the nostrils to maintain this immobilization and to promote internal coagulation, prevent septal hematomas and avert synechiae.

This conventional approach may actually cause edema and hematomas. When both internal packing and the external splint are applied, the venous capillaries are compressed, interfering with venous return and the healing process. Thus, blood is evacuated upward towards the eyelids and creates black eyes.

In addition, packing is aversive because of the resulting pain, headaches and nauseating odours (Tables 1 and 2). When the packing is removed, relief is immediate. 


\section{TABLE 1: Inconveniences arising from nasal packing following rhinoplasty}

Pain

Airway obstruction

Bleeding on removal

Unpleasant odour

Headaches

Ecchymosis + swelling of eyelids

\section{TABLE 2: Complications (theoretical) of nasal packing}

Sinusitis

Rhinitis

Otitis

Asphyxia by nocturnal aspiration

Septic shock

By using small instruments and reducing trauma to nasal tissues when performing rhinoplasty, we have been able to eliminate nasal packing and splint casts. General edema and ecchymosis of the eyelids is significantly decreased. Some of our patients can breathe through the nose with little difficulty within minutes after surgery, and there is no pain. We have treated many hundreds of cases without complications since 1982. This paper describes the technique in detail.

\section{TECHNIQUE}

Most cases are done under narcolepsy. When the patient arrives, we administer 15 $\mathrm{mg}$ diazepam orally. Once installed on the operating table and drawings are done, we generally inject $2 \mathrm{~mL}$ of fentanyl intravenously over a period of 3 mins. Not only does it sedate the patient but its strong analgesic effect facilitates infiltration of the nose which must be undertaken within 10 mins. More fentanyl can be given to heavier patients. Occasionally, at the time of osteotomy, we administer a further $1 \mathrm{~mL}$ of fentanyl. This is done with an oximeter by monitors and staff trained in cardiopulmonary resuscitation. We have used narcolepsy without any incidents.

Undermining of the dorsum is kept to a minimum, allowing a rasp to be inserted. A narrow osteotome is used with a narrow guard. Undermining of the skin or mucosa is avoided. We do not use a midline fracture because we want to maintain the naso-orbital line and keep the fractured bones stable. Indications for this manoeuvre are rare.

A 'green stick fracture' is performed by inserting the osteotome past the midpoint of the bone. We then raise the osteotome and rotate it internally, creating a green stick fracture with a very solid cephalic attachment.

With this technique, the cephalic part of the bone is not separated from the forehead bone. It will not narrow the radix which, in our experience, rarely requires narrowing. With this technique, in addition to maintaining naso-orbital continuity, we ensure that there is strong stability without the floating fragments of a conventional lateral bone fracture, or midline separation as when a medial osteotomy is performed. There is little chance that bone fragments can be displaced. They are relatively stable and 
do not require splinting or any other form of immobilization. Another advantage of this technique is that the osteotome will not transect the angular artery, the most common cause of excessive ecchymosis of the eyelids.

In cases with hypertrophied lower turbinates, we use bipolar cautery with good success. Unlike conventional turbinectomy, there is no need for packing.

We suture all the vestibular skin and mucosa, eliminating the risk of synechiae and septal hematomas. If we undermine the mucosa of the septum, we use ' $U$ ' type stitches to reapproximate the mucosa on the septum. A few Steri-Strips are applied to shape the skin and the underlying cartilage.

The outcome of a rhinoplasty procedure performed without splints or packing are illustrated in Figure 1, and the results from nearly 200 procedures presented in Table 3.
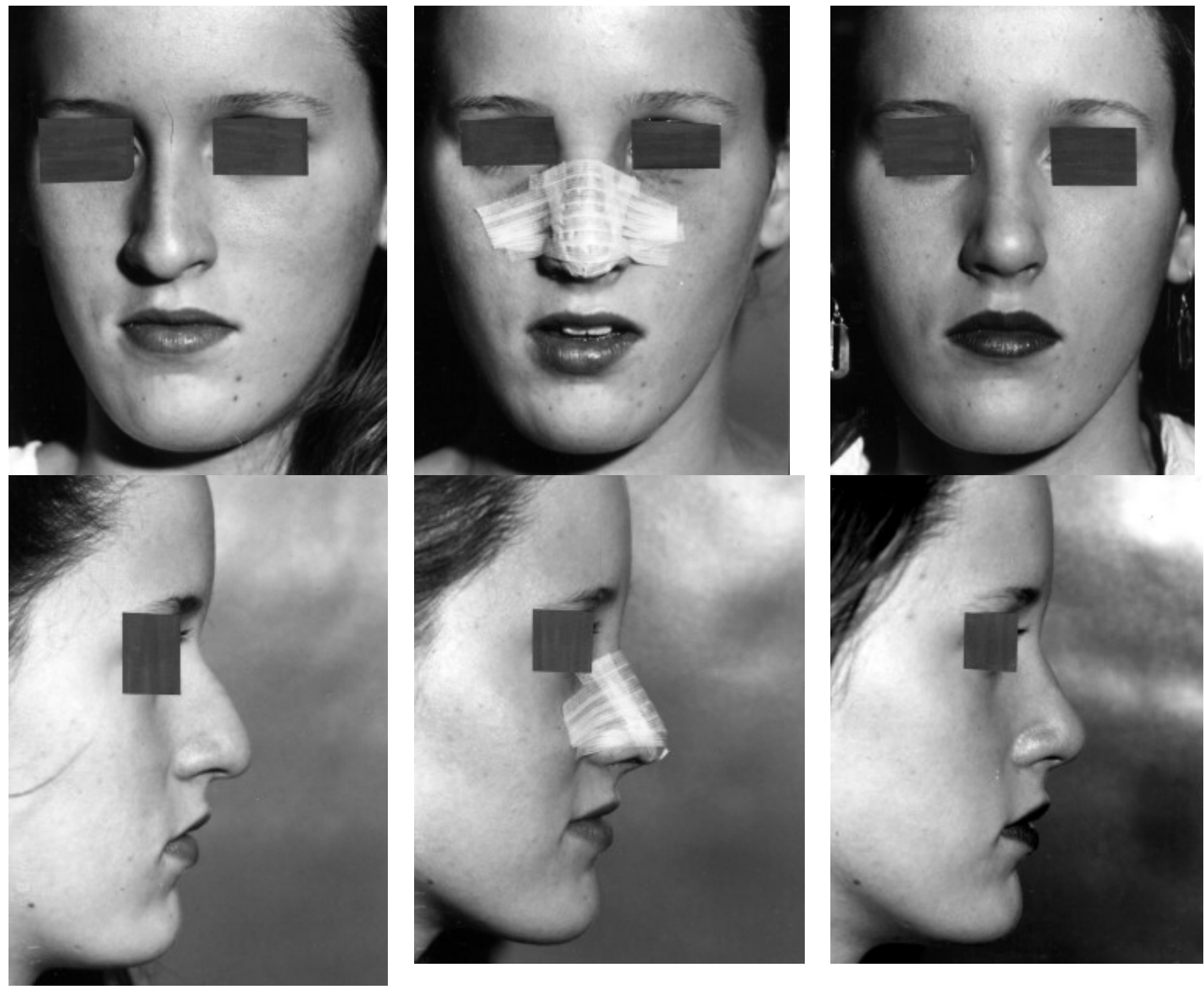

Figure 1) Results of rhinoplasty performed without splints or packing. (Top and bottom left) Preoperative presentation. (Top and bottom middle) Two days postoperative. (Top and bottom right) Two weeks postoperative 
TABLE 3: Review of 195 rhinoplasties performed without splints or packing

$\begin{array}{ll}\text { Number of patients with splint or cast } & 0\end{array}$

Number of patients with packing $\quad 0$

$\begin{array}{ll}\text { Number of cases with epistaxis } & 0\end{array}$

Number of patients requiring secondary osteotomy because the bones were not immobilized

Number of patients under local anaesthesia

Number of patients under general anaesthesia

\section{SUMMARY}

The procedure described here eliminates the need for splints and packing. It reduces swelling and ecchymosis of the eyelids. There is less risk of headache, rhinitis, sinusitis or otitis. Unpleasant odours do not occur. Since the removal of packing causes unnecessary bleeding in many cases, this complication is also eliminated.

We have never observed hemorrhages, septal hematoma or necrosis. We have not had a case of bone displacement resulting from absence of a splint or cast. Our patients feel comfortable, are free from pain and show minimal swelling. Some of them are able to breathe normally within minutes after surgery. The extent and instances of black eyes have been negligible

\section{REFERENCE}

Personal communications with Dr George Peck 